\title{
EXTRACTION OF RESONANCE PARAMETERS AND ROLE OF THE FINAL STATE INTERACTIONS*
}

\author{
IGOR I. STRAKOVSKY \\ Physics Department, The George Washington University \\ igor@gwu.edu \\ WILLIAM J. BRISCOE \\ Physics Department, The George Washington University \\ briscoe@gwu.edu \\ ALEXANDER E. KUDRYAVTSEV \\ Institute of Theoretical and Experimental Physics, Moscow, 117259 Russia and \\ Physics Department, The George Washington University \\ kudryavt@itep.ru \\ VLADIMIR E. TARASOV \\ Institute of Theoretical and Experimental Physics, Moscow, 117259 Russia \\ tarasov@itep.ru
}

Published 14 January 2014

\begin{abstract}
We present an overview of the SAID group effort to analyze new $\gamma n \rightarrow \pi^{-} p$ cross sections vs. the world database to get new multipoles and determine neutron electromagnetic couplings. The differential cross section for the processes $\gamma n \rightarrow \pi^{-} p$ was extracted from new measurements at CLAS and MAMI-B accounting for Fermi motion effects in the impulse approximation (IA) as well as NN- and $\pi N$-FSI effects beyond the IA. We evaluated results of several pion photoproduction analyses and compared $\pi N$ PWA results as a constraint for analyses of pion photoproduction data (Watson's theorem).
\end{abstract}

Keywords: FSI; PWA; Watson's Theorem.

PACS numbers: 13.60.Le, 13.30.-a

\section{Introduction}

An accurate evaluation of the electromagnetic (EM) couplings $N^{*}\left(\Delta^{*}\right) \rightarrow \gamma N$ from meson photoproduction data remains a paramount task in hadron physics. A wealth

*This is an Open Access article published by World Scientific Publishing Company. It is distributed under the terms of the Creative Commons Attribution 3.0 (CC-BY) License. Further distribution of this work is permitted, provided the original work is properly cited. 


\section{I. Strakovsky et al.}

of new data for meson photoproduction is becoming available from nuclear facilities worldwide. These measurements are now beginning to have a significant impact on both the resonance spectrum and its decay properties.

Here we focus on the single-pion production data and note that a complete solution requires couplings from both charged and neutral resonances, ${ }^{1,2}$ the latter requiring $\pi^{-} p$ and $\pi^{0} n$ photoproduction off a neutron target, typically a neutron bound in a deuteron target. Extraction of the two-body $\left(\gamma n \rightarrow \pi^{-} p\right.$ and $\gamma n \rightarrow$ $\left.\pi^{0} n\right)$ cross sections requires the use of a model-dependent nuclear correction, which mainly comes from final-state interactions (FSI). ${ }^{3}$ As a result, our knowledge of the neutral resonance couplings is less precise than that of the charged values for well-known low-laying baryons.

\section{Neutron-target Data for Pion Photoproduction}

In addition to being less precise, experimental data for neutron-target photoreactions are much less abundant than those utilizing a proton target, constituting only about $15 \%$ of the present SAID database. ${ }^{4}$ At low to intermediate energies, this lack of neutron-target data is partially compensated by experiments using pionic beams, e.g., $\pi^{-} p \rightarrow \gamma n$, as has been measured, for example, by the Crystal Ball Collab. at $\mathrm{BNL}^{5}$ for the inverse photon energy $\mathrm{E}_{\gamma}=285-689 \mathrm{MeV}$ and $\theta=41$ $-148^{\circ}$, where $\theta$ is the inverse production angle of pion in the CM frame. This process is free from complications associated with the deuteron target. However, the disadvantage of using the reaction $\pi^{-} p \rightarrow \gamma n$ is the 5 to 500 times larger cross sections for $\pi^{-} p \rightarrow \pi^{0} n \rightarrow \gamma \gamma n$, depending on $\mathrm{E}_{\gamma}$ and $\theta$.

Recently, we applied our FSI corrections ${ }^{6}$ to CLAS Collab. $\gamma d \rightarrow \pi^{-} p p$ data $\left(\mathrm{E}_{\gamma}=1050-2700 \mathrm{MeV} \text { and } \theta=32-157^{\circ}\right)^{7}$ to get elementary cross sections for $\gamma n \rightarrow \pi^{-} p .{ }^{8}$ The FSI correction factor for the CLAS kinematics was found to be small, $\Delta \sigma / \sigma<10 \%$. However, these new cross sections departed significantly from our predictions at the higher energies, and greatly modified the fit result, which allows to determine new neutron couplings (see below).

In our recent study, ${ }^{9}$ we addressed to the differential cross section measurements for $\gamma n \rightarrow \pi^{-} p$ in the $\Delta$-isobar region. The data came from MAMI-B $\left(\mathrm{E}_{\gamma}=301-\right.$ $455 \mathrm{MeV}$ and $\left.\theta=58-141^{\circ}\right) .{ }^{10}$ At energies dominated by the $\Delta$-resonance, the isospin $3 / 2$ multipoles are constrained by extensive studies performed using proton targets. The forward peaking structure is due largely to the Born contribution, which is well known. As a result, one would expect models to give predictions within a tight range, which is confirmed in Fig. 1.

\section{FSI for $\gamma n \rightarrow \pi^{-} p$}

We extract the $\gamma n \rightarrow \pi^{-} p$ cross section on free nucleon from the deuteron data in the quasi-free (QF) kinematic region of the $\gamma d \rightarrow \pi^{-} p p$ reaction with fast knocked-out proton and slow proton-spectator assumed not to be involved in the pion production process. In this, so-called Impulse Approximation (IA), ${ }^{13}$ the reaction mechanism 


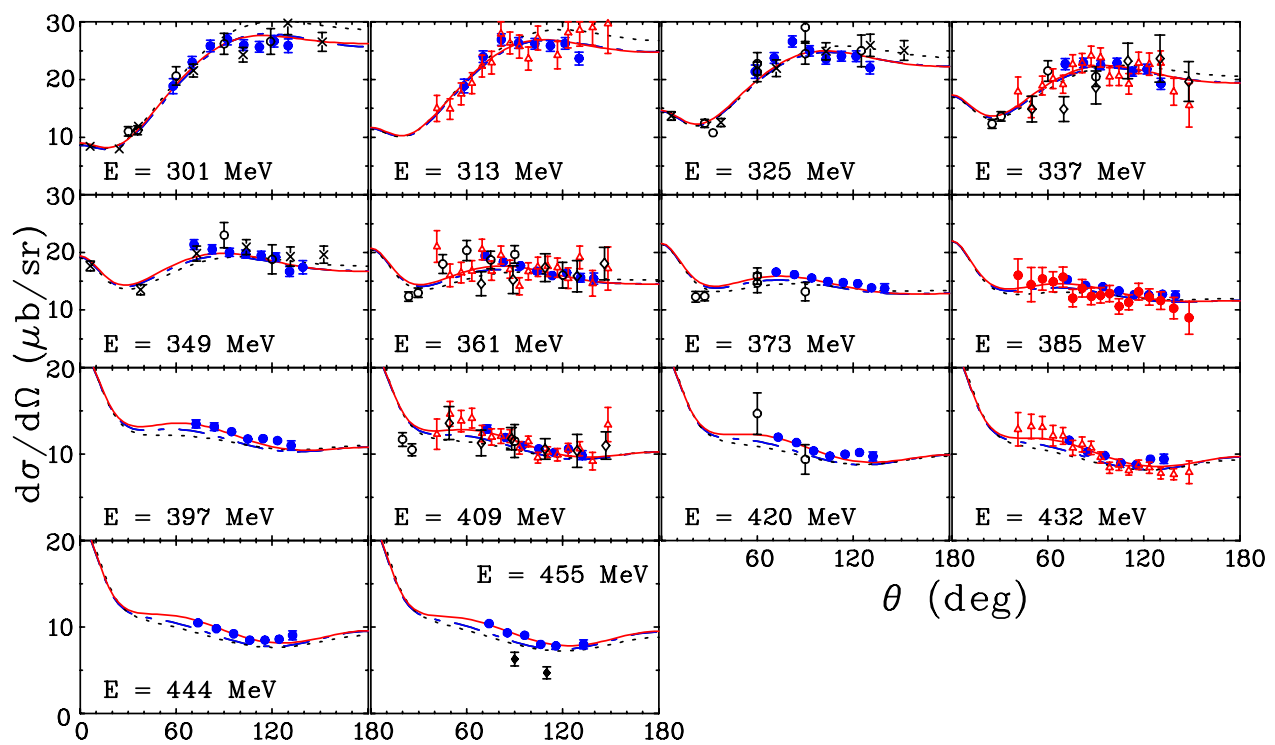

Fig. 1. Differential cross sections for $\gamma n \rightarrow \pi^{-} p$ as a function of $\theta$, where $\theta$ is the pion production angle in the CM frame. The MAMI-B data ${ }^{9}$ (filled circles) are shown for 14 energy bins. The rest of shown data came from hadronic facilities. ${ }^{4}$ MAMI-B data including in the SAID recent fit (solid lines). Dash-dotted (dotted) lines correspond to the predictions for the SAID SN11 ${ }^{11}$ (MAID07 ${ }^{12}$ ) solution.

corresponds to the diagram in Fig. 2(a). There are 2 critical factors to be taken into account when using this approach: (i) the neutron is bound and (ii) there are NN- and $\pi N$-FSI effects. Item (i) means that the effective mass of the neutron is not equal to the mass of the free neutron. In our former analyses, ${ }^{8,9}$ the $\gamma n \rightarrow \pi^{-} p$ amplitude for a given $\mathrm{E}_{\gamma}$ and $\mathrm{CM}$ pion production angle $\theta$ is assumed to be the same as on a free neutron at rest. That is why the cross section obtained should be considered as an average over energies around $\mathrm{E}_{\gamma}$. The size of the averaging region is determined by a smearing of the energy owing to the Fermi-motion in the deuteron. The typical scale here is $20 \mathrm{MeV}$ in energy.

Item (ii) corresponds to the inclusion of the FSI corrections. Their leading terms correspond to Feynman diagrams shown on Fig. 2(b,c). Determinations of the $\gamma d \rightarrow$ $\pi^{-} p p$ differential cross section, with the FSI taken into account (all the diagrams on

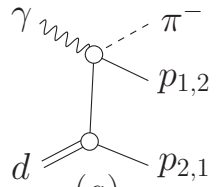

(a)

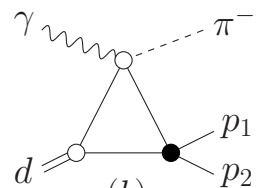

(b)

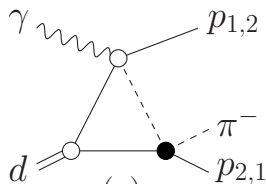

(c)

Fig. 2. Feynman diagrams for the leading terms of the $\gamma d \rightarrow \pi^{-} p p$ amplitude. (a) IA, (b) $p p-$ FSI, and (c) $\pi$ N-FSI. Filled black circles show FSI vertices. Wavy, dashed, solid, and double lines correspond to the photons, pions, nucleons, and deuterons, respectively. 


\section{I. Strakovsky et al.}

Fig. 2, were included) were done recently for the $\mathrm{CLAS}^{8}$ and MAMI-B ${ }^{9} \gamma d \rightarrow \pi^{-} p p$ data. The SAID phenomenological amplitudes for $\gamma N \rightarrow \pi N,{ }^{14}$ NN-elastic, ${ }^{15}$ and $\pi N$-elastic ${ }^{16}$ were used as inputs to calculate the diagrams in Fig. 2. The Bonn potential $^{17}$ was used for the deuteron description.

\section{Impact of CLAS $\gamma n \rightarrow \pi^{-} p$ data for EM Neutron Couplings, $\mathrm{S}=0$ and $\mathrm{I}=1 / 2$}

We have included the new cross sections from the CLAS experiment ${ }^{8}$ in a number of multipole analyses covering incident photon energies up to $2.7 \mathrm{GeV}$, using the full SAID database, ${ }^{4}$ in order to gauge the influence of these measurements, as well as their compatibility with previous experiments. The solution, GB12, uses the same fitting form as our recent SN11 solution. ${ }^{11}$ A second fit, GZ12, instead used the recently proposed form based on a unified Chew-Mandelstam parametrization of the GW DAC fits to both $\pi N$ elastic scattering and photoproduction. ${ }^{18}$ Resonance couplings found for both GB12 and GZ12 are close to each other.

Table 1 shows that the new GB12 $n A_{1 / 2}$ and $n A_{3 / 2}$ helicities sometimes have a significant deviation from the previous SAID SN11 ${ }^{11}$ determination and PDG12 ${ }^{20}$ values, e.g., for $N(1650) 1 / 2^{-}, N(1675) 5 / 2^{-}$, and $N(1680) 5 / 2^{+}$. While recent BnGa13 ${ }^{19}$ got a similar results vs. GB12 and PDG12, ${ }^{20}$ e.g., $N(1650) 1 / 2^{-}$ and $N(1680) 5 / 2^{+}$. Let us stress that both SAID GB12 and BnGa13 used the same (almost) data to fit them.

\section{Watson's Theorem}

The connection between scattering and decay processes provides a solid theoretical ground for describing some hadronic effects - Watson's theorem. ${ }^{1}$ For pion photoproduction, isospin amplitudes have to satisfy Watson's theorem below the

Table 1. Neutron helicity amplitudes $n A_{1 / 2}$ and $n A_{3 / 2}$ (in $\left[(\mathrm{GeV})^{-1 / 2} \times 10^{-3}\right]$ units) from the GB12 ${ }^{8}$ solution (first row), previous $\mathrm{SN}_{11}{ }^{11}$ solution (second row), BnGa13 ${ }^{19}$ solution (third row), and average values from the PDG12 20 (forth row).

\begin{tabular}{|cc|ccc|}
\hline Resonance & $n A_{1 / 2}$ & Resonance & $n A_{1 / 2}$ & $n A_{3 / 2}$ \\
\hline$N(1535) 1 / 2^{-}$ & $-58 \pm 6$ & $N(1520) 3 / 2^{-}$ & $-46 \pm 6$ & $-115 \pm 5$ \\
& $-60 \pm 3$ & & $-47 \pm 2$ & $-125 \pm 2$ \\
& $-93 \pm 11$ & & $-49 \pm 8$ & $-113 \pm 12$ \\
$N(1650) 1 / 2^{-}$ & $-46 \pm 27$ & & $-59 \pm 9$ & $-139 \pm 11$ \\
& $-40 \pm 10$ & $N(1675) 5 / 2^{-}$ & $-58 \pm 2$ & $-80 \pm 5$ \\
& $25 \pm 20$ & & $-42 \pm 2$ & $-60 \pm 2$ \\
$N(1440) 1 / 2^{+}$ & $-15 \pm 21$ & & $-60 \pm 7$ & $-88 \pm 10$ \\
& $48 \pm 4$ & $N(1680) 5 / 2^{+}$ & $-43 \pm 12$ & $-58 \pm 13$ \\
& $45 \pm 15$ & & $50 \pm 4$ & $-29 \pm 2$ \\
$43 \pm 12$ & & $34 \pm 6$ & $-47 \pm 2$ \\
$40 \pm 10$ & & $29 \pm 10$ & $-33 \pm 9$ \\
\hline
\end{tabular}


$2 \pi$-threshold allowing for a smooth departure from constraint at high energies.

$$
\operatorname{atan}\left(\frac{\operatorname{ImA} A_{l}}{\operatorname{ReA}_{l}}\right)=\delta_{l}(\pi N) .
$$

Above $2 \pi$-threshold, the rule may still be true, if inelasticity of the corresponding $\pi N$-elastic amplitude is small (as, e.g., for $P_{33}$.)

We evaluated the results of several pion photoproduction analyses such as SAID,${ }^{16,18}$ MAID, ${ }^{12}$ EBAC, ${ }^{21}$ Giessen, ${ }^{22}$ and $\mathrm{BnGa}^{23}$ and compared $\pi N$ PWA results as a constraint for analyses of pion photoproduction data. Most of the pion photoproduction analyses use SAID $\pi N$ outcome or its modification as input.

Our short summary is (i) Phases coming from $\pi N$ amplitudes of different analyses are consistent; (ii) Some phases coming from different pion photoproduction analyses are consistent with each other and phases coming from $\pi N$ amplitudes: $\mathrm{I}=3 / 2: p E 0^{+}, p E 1^{+}$, and $p M 1^{+}$; (iii) Some phases coming from different pion photoproduction analyses are inconsistent with each other and phases coming from $\pi N$ amplitudes: $\mathrm{I}=3 / 2: p M 1^{-}, p E 2^{-}, p M 2^{-}, p E 2^{+}$, and $p M 2^{+} ; \mathrm{I}=1 / 2: p E 0^{+}, p M 1^{-}$, $p E 1^{+}, p M 1^{+}, p E 2^{-}, p M 2^{-}, p E 2^{+}$, and $p M 2^{+}$; (iv) Some phases coming from $E$ and $M$ multipoles are inconsistent with each other and phases coming from $\pi N$ amplitudes. See, for instance, Fig. 3, which shows one worse case for BnGa.
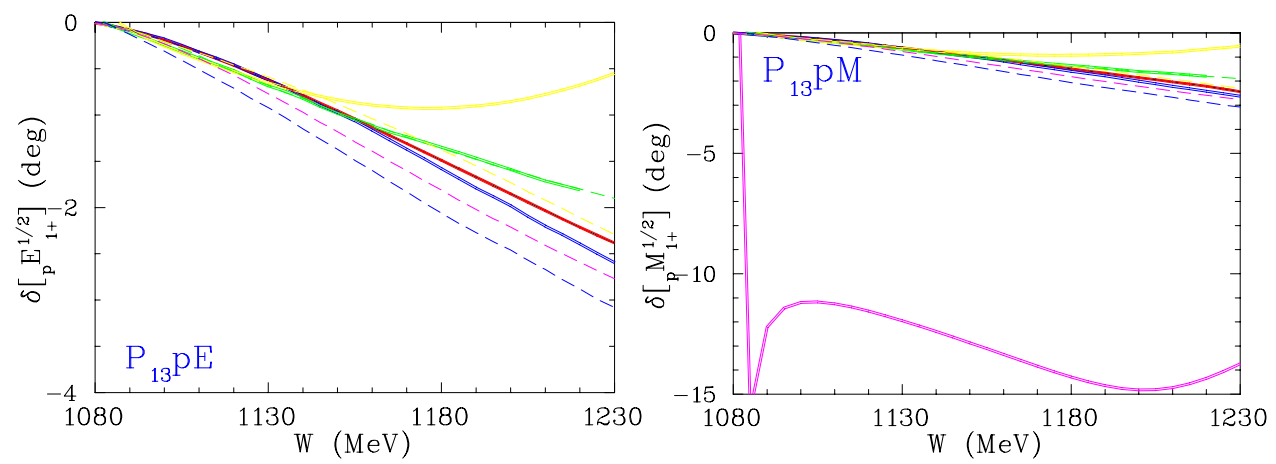

Fig. 3. $p E_{1+}^{1 / 2}$ (left) and $p M_{1+}^{1 / 2}$ (right) multipoles. Dashed (solid) lines show phases came from $\pi N$ elastic scattering (pion photoproduction). Double solid lines shown BnGa results.

\section{Very Strange Project for CLAS12}

This Section is written upon request of the Chairman of the Nstar 2013 Workshop, Eulogio Oset.

The first goals of a Very Strange Project for JLab CLAS12 ${ }^{24}$ are cross section measurements for $\gamma p \rightarrow \Omega^{-} K^{+} K^{+} K^{0}$ which is still unknown, and then to study a mechanism of the $\Omega^{-}$photoproduction which should be quite specific, since it is the first baryon with constituents none of which could come from the target proton. The further physics goals are mass splitting measurements of cascades, study of excited 


\section{I. Strakovsky et al.}

cascade resonances to determine a spin-parity, and polarization measurements of cascades. Assuming the $\Omega^{-}$cross section in a photoproduction on the proton close to the threshold as $0.3 \mathrm{nb}$, we are expecting to have $5 \mathrm{k} \Omega \mathrm{s}$ and simultaneously $1.5 \mathrm{M} \Xi$ s during an 80 day run period with CLAS12 and Forward Tagger facilities. We hope that it may allow to determine not only $\sigma^{\text {tot }}$ of $\Omega^{-}$photoproduction but $d \sigma / d \Omega$ as well.

\section{Acknowledgments}

This work was supported in part by the U. S. Department of Energy Grant No. DE-FG02-99ER41110. A. E. K. thanks grant NS-3172.2012.2 for partial support.

\section{References}

1. K. M. Watson, Phys. Rev. 95, 228 (1954).

2. R. L. Walker, Phys. Rev. 182, 1729 (1969).

3. A. B. Migdal, JETP 1, 2 (1955); K. M. Watson, Phys. Rev. 88, 1163 (1952).

4. Institute of Nuclear Studies of GW Database (W. J. Briscoe, I. I. Strakovsky, and R. L. Workman); http://gwdac.phys.gwu.edu/analysis/pr_analysis.html .

5. Crystal Ball Collab. (A. Shafi et al.), Phys. Rev. C 70, 035204 (2004).

6. V. E. Tarasov et al., Phys. Rev. C 84, 035203 (2011).

7. CLAS Collab. (W. Chen et al.), Phys. Rev. Lett. 103, 012301 (2009).

8. W. Chen et al., Phys. Rev. C 86, 015206 (2012).

9. W. J. Briscoe et al., Phys. Rev. C 86, 065207 (2012).

10. GDH and A2 Collab. (J. Ahrens, et al.), Eur. Phys. J. A 44, 189 (2010).

11. R. Workman et al., Phys. Rev. C 85, 025201 (2012).

12. D. Drechsel et al., Eur. Phys. J. A 34, 69 (2007); L. Tiator, private communication, 2008.

13. G. Chew and M. Goldberger, Phys. Rev. 87, 778 (1952).

14. CLAS Collab. (M. Dugger et al.) Phys. Rev. C 76, 025211 (2007).

15. R. A. Arndt et al. Phys. Rev. C 76, 025211 (2007).

16. R. A. Arndt et al. Phys. Rev. C 74, 045205 (2006).

17. R. Machleidt, K. Holinde, and C. Elster, Phys. Rept. 140, 1 (1987).

18. R. Workman et al., Phys. Rev. C 86, 015202 (2012).

19. A. Anisovich et al., Eur. Phys. J. A 49, 67 (2013).

20. Particle Data Group (J. Beringer et al.), Phys. Rev. D 86, 010001 (2012).

21. B. Julia-Diaz et al., Phys. Rev. C 76, 065201 (2007); T. Sato et al., Phys. Rev. C 54, 2660 (1996).

22. V. Shklyar et al., Phys. Rev. C 71, 055206 (2005); V. Shklyar, private communication, 2007.

23. A. Anisovich et al., Eur. Phys. J. A 48, 15 (2012); A. Sarantsev, private communication, 2009.

24. Photoproduction of the very strangest baryons on the proton target in CLAS12, The Very Strange Collab. (L. Guo et al.), JLab Proposal E12-11-005a, Newport News, VA, USA, 2013. 